\title{
A Deflation Approach to Direction of Arrival Estimation for Symmetric Uniform Linear Array
}

\author{
$\mathrm{Xu} \mathrm{Xu,} \mathrm{Zhongfu} \mathrm{Ye,} \mathrm{Yufeng} \mathrm{Zhang,} \mathrm{and} \mathrm{Chunqi} \mathrm{Chang}$
}

\begin{abstract}
A novel deflation approach to direction of arrival (DOA) estimation for symmetric uniform linear array is proposed in this letter to cope with the scenario where both uncorrelated sources and coherent sources are presented. The uncorrelated sources are first estimated using conventional subspace methods, and then their effect is eliminated by two deflation methods: one exploits the symmetric configuration of the array, and the other utilizes oblique projection. After deflation, a Toeplitz matrix is constructed for DOA estimation of the remaining coherent sources. The number of sources resolved by our approach can exceed the number of array elements. Simulation results demonstrate the effectiveness and efficiency of our proposed methods.
\end{abstract}

Index Terms-Coherent sources, deflation, direction of arrival (DOA), oblique projection, symmetric uniform linear array, Toeplitz matrix.

\section{INTRODUCTION}

D IRECTION of arrival (DOA) estimation of multiple narrowband sources is a major research issue in array signal processing. Many high-resolution DOA estimation methods, such as the famous MUSIC and ESPRIT, have been developed over the years [1]-[3]. However, in real environments, those high-resolution methods may fail to work when there are highly correlated or coherent sources due to multipath propagation, since the methods inherently require the sources to be uncorrelated or lowly correlated. Some preprocessing techniques [4], [5] can be used to decorrelate the coherent source signals, but they usually reduce the effective number of array elements. In [5], a Toeplitz matrix reconstructed from the covariance matrix is used to realize a better DOA estimation no matter whether the sources are coherent or not. A main disadvantage is that in this approach the number of sources can be resolved is at most half of the number of array elements.

In this letter, a deflation approach is proposed for DOA estimation when there are both uncorrelated sources and coherent sources. Two deflation methods are developed. The uncorrelated sources are estimated first, and then they are eliminated by exploiting the symmetric property of array elements or using oblique projection [6] so that the coherent sources can be estimated by the method proposed in [5]. In this approach,

Manuscript received July 7, 2006; revised October 3, 2006.

$\mathrm{X}$. Xu, Z. Ye, and Y. Zhang are with the Department of Electronic Engineering and Information Science, University of Science and Technology of China, Hefei 230027, P.R. China (e-mail: xxu@ustc.edu.cn; yezf@ustc.edu.cn; myth@mail.ustc.edu.cn).

C. Chang is with the Department of Electrical and Electronic Engineering, The University of Hong Kong, Hong Kong (e-mail: cqchang@eee.hku.hk).

Digital Object Identifier 10.1109/LAWP.2006.886304 the number of resolvable sources can be even greater than the number of array elements.

\section{FoRMULATION OF THE PROBLEM}

Consider a number of $K$ narrowband far-field sources impinging on a symmetric uniform linear array with $M=2 N+1$ array elements, where the distance between adjacent elements is equal to half of the wavelength. Assume that there are coherent sources due to multipath propagation. Group the coherent sources together and denote the number of groups as $P$. In the $i$ th group, assume the coherent source coming from the direction $\theta_{i l}$ is corresponding to the $l$ th multipath propagation of the source $S_{i}(t)$ with power $\sigma_{i}^{2}$, and $l=1, \ldots, L_{i}$. The sources within each group are coherent from each other and uncorrelated to the sources in a different group. Denote the total number of coherent sources as $L=\sum_{i=1}^{P} L_{i}$, and assume the remaining $D=K-L$ sources, $S_{i}(t)$ coming from direction $\theta_{i}$ with signal power $\sigma_{i}^{2}$ for $i=L+1, \ldots, K$, are uncorrelated to each other and also to the coherent sources. Let the index of the central element of the array to be 0 , the $M \times 1$ array output vector $x(t)$ is then given by

$$
\begin{aligned}
x(t) & =\left[x_{-N}(t), \ldots, x_{0}(t), \ldots, x_{N}(t)\right]^{T} \\
& =\sum_{i=1}^{P} \sum_{l=1}^{L_{i}} \boldsymbol{\alpha}\left(\theta_{i l}\right) \rho_{i l} s_{i}(t)+\sum_{i=L+1}^{K} \boldsymbol{\alpha}\left(\theta_{i}\right) s_{i}(t)+n(t) \\
& =\boldsymbol{A}_{c} \boldsymbol{s}_{c}(t)+\boldsymbol{A}_{n} \boldsymbol{s}_{n}(t)+n(t)=\boldsymbol{A} \boldsymbol{s}(t)+\boldsymbol{n}(t)
\end{aligned}
$$

where $\boldsymbol{a}(\theta)$ is the steering vector, $\rho_{i l}$ is the complex fading coefficient of the $l$ th coherent source in the $i$ th group, $\rho_{i}=\left[\rho_{i 1}, \ldots, \rho_{i L_{i}}\right]^{T}, \boldsymbol{A}_{i}=\left[\boldsymbol{\alpha}\left(\theta_{i 1}\right), \ldots, \boldsymbol{\alpha}\left(\theta_{i L_{i}}\right)\right]$, $\boldsymbol{A}_{c}=\left[\boldsymbol{A}_{1} \boldsymbol{\rho}_{1}, \ldots, \boldsymbol{A}_{P} \boldsymbol{\rho}_{P}\right], \boldsymbol{A}_{n}=\left[\boldsymbol{\alpha}\left(\theta_{L+1}\right), \ldots, \boldsymbol{\alpha}\left(\theta_{K}\right)\right]$, $\boldsymbol{s}_{c}(t)=\left[s_{1}(t), \ldots, s_{P}(t)\right]^{T}, \boldsymbol{s}_{n}(t)=\left[s_{L+1}(t), \ldots, s_{K}(t)\right]^{T}$, $\boldsymbol{A}=\left[\boldsymbol{A}_{c}, A_{n}\right], \boldsymbol{s}(t)=\left[\boldsymbol{s}_{c}^{T}(t), \boldsymbol{s}_{n}^{T}(t)\right]^{T}$, and $\boldsymbol{n}(t)$ is the noise vector with the power of each entry equal to $\sigma_{n}^{2}$. By assumption, the entries of $\boldsymbol{s}_{c}(t), \boldsymbol{s}_{n}(t)$ and $\boldsymbol{n}(t)$ are zero mean wide-sense stationary random processes and are uncorrelated to each other.

From (1), we can obtain the array covariance matrix

$$
\begin{aligned}
\boldsymbol{R}_{x} & =E\left\{\boldsymbol{x}(t) \boldsymbol{x}^{H}(t)\right\}=\boldsymbol{A R}_{s} \boldsymbol{A}^{H}+\sigma_{n}^{2} \boldsymbol{I}_{M} \\
& =\boldsymbol{A}_{c} \boldsymbol{R}_{c} \boldsymbol{A}_{c}^{H}+\boldsymbol{A}_{n} \boldsymbol{R}_{n} \boldsymbol{A}_{n}^{H}+\sigma_{n}^{2} \boldsymbol{I}_{M}
\end{aligned}
$$

where $\boldsymbol{R}_{c}$ and $\boldsymbol{R}_{n}$ are the covariance matrix of $\boldsymbol{s}_{c}(t)$ and $\boldsymbol{s}_{n}(t)$, respectively, $\boldsymbol{I}_{M}$ represents the $M \times M$ identity matrix, and $\boldsymbol{R}_{s}=\operatorname{diag}\left\{\boldsymbol{R}_{c}, \boldsymbol{R}_{n}\right\}$ which is block diagonal. Due to the assumption of uncorrelatedness, it comes that $\boldsymbol{R}_{c}=\operatorname{diag}\left\{\sigma_{1}^{2}, \ldots, \sigma_{P}^{2}\right\}$ and $\boldsymbol{R}_{n}=\operatorname{diag}\left\{\sigma_{L+1}^{2}, \ldots, \sigma_{K}^{2}\right\}$. 


\section{DOA ESTIMATION}

\section{A. Estimation of the Uncorrelated Sources}

We first estimate the DOAs of the uncorrelated sources. In (2), each group of coherent sources can be considered as an equivalent virtual source. If $D+P<M$, the eigendecomposition of $\boldsymbol{R}_{x}$ can be written in the form

$$
\boldsymbol{R}_{x}=U_{s} \boldsymbol{\Sigma}_{s} \boldsymbol{U}_{s}^{H}+\boldsymbol{U}_{n} \boldsymbol{\Sigma}_{n} \boldsymbol{U}_{n}^{H}
$$

where $U_{s}$ is a $M \times(D+P)$ unitary matrix whose columns are the eigenvectors corresponding to the $D+P$ largest eigenvalues, while the columns of $\boldsymbol{U}_{n}$ are the eigenvectors corresponding to the $M-D-P$ smallest eigenvalues. The columns of $\boldsymbol{U}_{s}$ span the signal subspace which is spanned also by the columns of $\boldsymbol{A}_{n}$ and $\boldsymbol{A}_{c}$ jointly, and the signal subspace is orthogonal to the noise subspace spanned by the columns of $\boldsymbol{U}_{n}$ [3]. Therefore,

$$
g(\theta) \triangleq\left|\boldsymbol{\alpha}^{H}(\theta) \boldsymbol{U}_{n}\right|^{2}=0 \text { when } \theta=\theta_{i}, \quad i=L+1, \ldots, K .
$$

Thus, the DOAs of the uncorrelated sources are corresponding to the peaks of $1 / g(\theta)$. Then the matrix $\boldsymbol{A}_{n}$ can be calculated from the estimated DOAs.

\section{B. Estimation of the Coherent Sources: Method 1}

This method exploits the symmetric configuration of the array. As in [5], define a matrix $\boldsymbol{R}$ and its entries $r(m, k)$, $m, k=-N, \ldots, 0, \ldots, N$, as

$$
\begin{aligned}
\boldsymbol{R} \triangleq & \boldsymbol{A} \boldsymbol{R}_{s} \boldsymbol{A}^{H}=\boldsymbol{R}_{x}-\sigma_{n}^{2} \boldsymbol{I}_{M} \\
= & \boldsymbol{U}_{s}\left(\boldsymbol{\Sigma}_{s}-\sigma_{n}^{2} \boldsymbol{I}_{D+P}\right) \boldsymbol{U}_{s}^{H} \\
r(m, k)= & \sum_{i=1}^{P} \sum_{l=1}^{L_{i}} b_{m, i}^{l} e^{j \pi k \sin \theta_{i l}} \\
& +\sum_{i=L+1}^{K} d_{m, i} e^{j \pi k \sin \theta_{i}}
\end{aligned}
$$

where $b_{m, i}^{l}=\sigma_{i}^{2} \rho_{i l}^{*} \sum_{p=1}^{L_{i}} \rho_{i p} e^{-j \pi m \sin \theta_{i p}}$ and $d_{m, i}=$ $\sigma_{i}^{2} e^{-j \pi m \sin \theta_{i}}$.

Then for $m, k=-N, \ldots, 0, \ldots, N$, define

$$
g(m, k)=r(m, k)-r^{*}(-m,-k) .
$$

From (6), $g(m, k)$ can be further rewritten as

$$
g(m, k)=\sum_{i=1}^{P} \sum_{l=1}^{L_{i}} q_{m, i}^{l} e^{j \pi k \sin \theta_{i l}}
$$

where $q_{m, i}^{l}=\sigma_{i}^{2} \sum_{p=1}^{L_{i}}\left(\rho_{i l}^{*} \rho_{i p}-\rho_{i l} \rho_{i p}^{*}\right) e^{-j \pi m \sin \theta_{i p}}$. Then, only coherent components remain in $g(m, k)$. Construct the following Toeplitz matrix as shown in (9)

$$
\begin{aligned}
\boldsymbol{G}(m) & =\left[\begin{array}{ccc}
g(m, 0), & g(m, 1), \ldots, & g(m, N) \\
g(m,-1), & g(m, 0), \ldots, & g(m, N-1) \\
\vdots & \vdots & \vdots \\
g(m,-N), & g(m,-N+1), \ldots, & g(m, 0)
\end{array}\right] \\
& =\boldsymbol{B} D(m) \boldsymbol{B}^{H}
\end{aligned}
$$

where $\boldsymbol{D}(m)=\operatorname{diag}\left\{q_{m, 1}^{1}, \ldots, q_{m, 1}^{L_{1}}, \ldots, q_{m, P}^{1}, \ldots, q_{m, P}^{L_{P}}\right\}$, $\boldsymbol{B}=\boldsymbol{J}\left[\boldsymbol{A}_{1}, \ldots, \boldsymbol{A}_{P}\right]$, and $\boldsymbol{J}=\left[\boldsymbol{O}_{(N+1) \times N}, \boldsymbol{I}_{N+1}\right]$. If $L \leq N$, from (8) and (9), the rank of $G(m)$ is generally $L$ for nontrivial cases. Applying high-resolution direction finding methods, such as MUSIC or ESPRIT, to $\boldsymbol{G}(m)$, we can obtain the DOA estimates of the coherent sources.

\section{Estimation of the Coherent Sources: Method 2}

This second deflation method utilizes oblique projection. According to the definition [6], an oblique projection $\boldsymbol{E}_{\boldsymbol{A}_{n} \boldsymbol{A}_{c}}$ is a nonorthogonal projection whose range is spanned by $\boldsymbol{A}_{n}$ and whose null space is spanned by $\boldsymbol{A}_{c}$, with the following properties:

$$
\boldsymbol{E}_{\boldsymbol{A}_{n} \boldsymbol{A}_{c}}^{2}=\boldsymbol{E}_{\boldsymbol{A}_{n} A_{c}}, \quad \boldsymbol{E}_{\boldsymbol{A}_{n} \boldsymbol{A}_{c}} \boldsymbol{A}_{n}=\boldsymbol{A}_{n}, \quad \boldsymbol{E}_{\boldsymbol{A}_{n} \boldsymbol{A}_{c}} \boldsymbol{A}_{c}=0 .
$$

We can see that while it nulls out $A_{c}$, it has no effect on $A_{n}$. The oblique projection can be obtained by

$$
\boldsymbol{E}_{\boldsymbol{A}_{n} \boldsymbol{A}_{c}}=\boldsymbol{A}_{n}\left(\boldsymbol{A}_{n}^{H} \boldsymbol{P}_{\boldsymbol{A}_{c}^{\perp}}^{\perp} \boldsymbol{A}_{n}\right)^{-1} \boldsymbol{A}_{n}^{H} \boldsymbol{P}_{\boldsymbol{A}_{c}}^{\perp}
$$

where $\boldsymbol{P}_{\boldsymbol{A}}^{\perp}$ denotes the orthogonal complement of the subspace spanned by the columns of $\boldsymbol{A}_{c}$. With the constructed $\boldsymbol{E}_{\boldsymbol{A}_{n}} \boldsymbol{A}_{c}$, we can now define

$$
\boldsymbol{H} \triangleq\left(\boldsymbol{I}_{M}-\boldsymbol{E}_{\boldsymbol{A}_{n} \boldsymbol{A}_{c}}\right) \boldsymbol{R}\left(\boldsymbol{I}_{M}-\boldsymbol{E}_{\boldsymbol{A}_{n} \boldsymbol{A}_{c}}\right)^{H} .
$$

Then, only coherent components remain in $\boldsymbol{H}$ since

$$
\boldsymbol{H}=\boldsymbol{A}_{c} \boldsymbol{R}_{c} \boldsymbol{A}_{c}^{H} .
$$

Similarly as in (8), the entries of $\boldsymbol{H}$ can be written as

$$
h(m, k)=\sum_{i=1}^{P} \sum_{l=1}^{L_{i}} b_{m, i}^{l} e^{j \pi k \sin \theta_{i l}} .
$$

Then we construct the following Toeplitz matrix as shown in (15)

$$
\begin{aligned}
\boldsymbol{F}(m) & =\left[\begin{array}{ccc}
h(m, 0), & h(m, 1), \ldots, & h(m, N) \\
h(m,-1), & h(m, 0), \ldots, & h(m, N-1) \\
\vdots & \vdots & \vdots \\
h(m,-N), & h(m,-N+1), \ldots, & h(m, 0)
\end{array}\right] \\
& =\boldsymbol{B} V(m) \boldsymbol{B}^{H}
\end{aligned}
$$

where $\boldsymbol{V}(m)=\operatorname{diag}\left\{b_{m, 1}^{1}, \ldots, b_{m, 1}^{L_{1}}, \ldots, b_{m, P}^{1}, \ldots, b_{m, P}^{L_{P}}\right\}$. Obviously, the matrix $\boldsymbol{F}(m)$ is also of rank $L$ in general. Similar as discussed in Section III-B, we can get the DOA estimates of the coherent sources by applying ESPRIT to $\boldsymbol{F}(m)$

There is a difficulty in obtaining $\boldsymbol{E}_{\boldsymbol{A}_{n} \boldsymbol{A}_{c}}$ by (11) because $\boldsymbol{P}_{\boldsymbol{A}_{c}}^{\perp}$ cannot be estimated directly without the knowledge of $\boldsymbol{A}_{c}$. As in [7], here we can use an alternative computation equation when $D+P<M$

$$
\boldsymbol{E}_{A_{n} \boldsymbol{A}_{c}}=\boldsymbol{A}_{n}\left(\boldsymbol{A}_{n}^{H} \boldsymbol{R}^{\#} \boldsymbol{A}_{n}\right)^{-1} \boldsymbol{A}_{n}^{H} \boldsymbol{R}^{\#}
$$

where \# denotes the operator of pseudoinverse, and $\boldsymbol{R}$ is computed by (5). The required $\boldsymbol{R}_{x}$ and $\sigma_{n}^{2}$ can be directly estimated through data samples. 
TABLE I

MAXIMUM NuMber of SOURCES RESOLVED FOR A 9-ELEMENT UNIFORM LINEAR ARRAY

\begin{tabular}{|c|c|c|c|c|}
\hline & \multicolumn{2}{|c|}{ Coherent sources } & \multirow{2}{*}{$\begin{array}{c}\text { Number of } \\
\text { uncorrelate } \\
\text { d sources }\end{array}$} & \multirow[b]{2}{*}{$\begin{array}{c}\text { Total } \\
\text { number }\end{array}$} \\
\hline & \begin{tabular}{|c|}
$\begin{array}{c}\text { Number } \\
\text { of groups }\end{array}$ \\
\end{tabular} & $\begin{array}{l}\text { Number in } \\
\text { each group }\end{array}$ & & \\
\hline \multirow{3}{*}{ Method 0} & 1 & 2 & 2 & 4 \\
\hline & 1 & 3 & 1 & 4 \\
\hline & 2 & 2 & 0 & 4 \\
\hline \multirow{2}{*}{ Method 1} & 1 & 4 & 7 & 11 \\
\hline & 2 & 2 & 6 & 10 \\
\hline \multirow{2}{*}{ Method 2} & 1 & 4 & 7 & 11 \\
\hline & 2 & 2 & 6 & 10 \\
\hline
\end{tabular}

\section{Discussion}

As stated in [5], we can construct the Toeplitz matrices for each $m=-N, \ldots, 0, \ldots, N$ and then take the average over these matrices to get a better estimation, for example $G=$ $\sum_{m} \boldsymbol{G}(m)=\boldsymbol{B} \sum_{m} \boldsymbol{D}(m) \boldsymbol{B}^{H}$. However, such average may be problematic for the estimation of uncorrelated sources using the method proposed in [5] since the amplitude of $\sum_{m} d_{m, i}=$ $\sigma_{i}^{2} \sum_{m} e^{-j \pi m \sin \theta_{i}}$, which is the response of the array, may be very small for some $\theta$ when the number of items exceeds 2 . Our approach uses conventional subspace methods at the first stage and thus has no such problem for the uncorrelated sources. In our second stage for the coherent sources, the effect of such average on $\sum_{m} q_{m, i}^{l}$ and $\sum_{m} b_{m, i}^{l}$ is similar to that proposed in [5]. Therefore, here we average over $m=0,1$ only.

\section{SiMUlation EXPERIMENTS AND DisCUSSION}

In this section, we illustrate the performance of the proposed methods. The number of array elements is 9 with $N=4$. The method proposed in [5] is referred as Method 0, and it is used to be compared to Method 1 and Method 2 described in Section III. Since the uncorrelated and the coherent sources are estimated at different stages, for comparison convenience, here Method 1 and Method 2 both include the estimation of the uncorrelated sources described in Section III-A. When using (4) to estimate the uncorrelated sources, the search is performed over $\left[-90^{\circ}, 90^{\circ}\right]$ with a step size $0.1^{\circ}$. The average Toeplitz matrices (over $m=0,1$ ), as discussed in Section III-D, are used for DOA estimation for the coherent sources by applying the ESPRIT algorithm. The number of snapshots used in the simulation is 500 . We perform 200 Monte Carlo trials for each experiment. The average root mean square error (rmse) of the DOA estimates is used as the performance index

$$
\text { rmse }=\sqrt{\frac{\sum_{n=1}^{200} \sum_{i=1}^{I}\left(\hat{\theta}_{i}(n)-\theta_{i}\right)^{2}}{200 I}}
$$

where $\hat{\theta}_{i}(n)$ is the estimate of $\theta_{i}$ for the $n$th Monte Carlo trial, and $I$ is the number of all the uncorrelated or all the coherent sources. Note that here we use different indices for uncorrelated sources and coherent sources, because they are estimated at different stages.

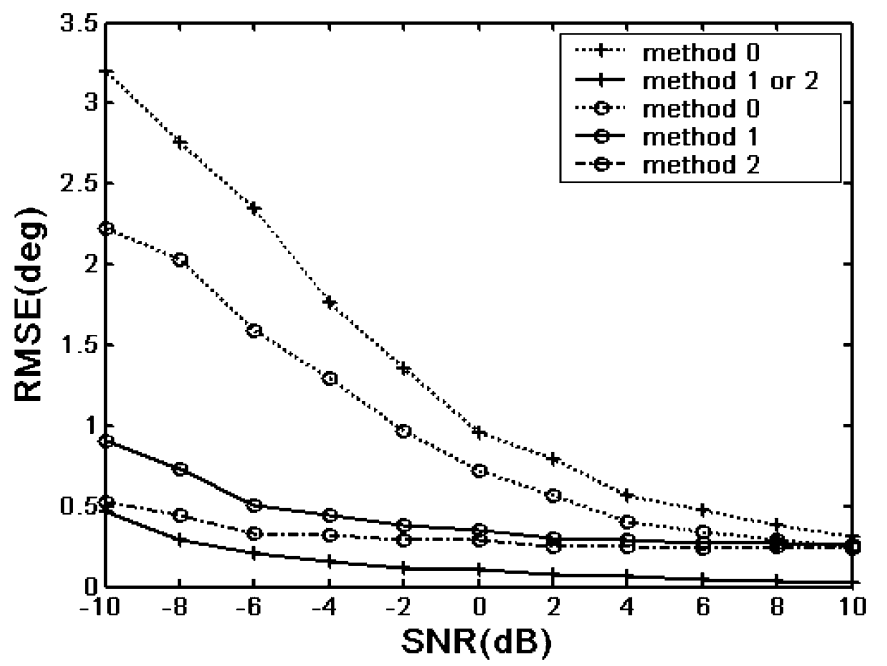

Fig. 1. rmse of the DOA estimates versus input SNR for uncorrelated $(+)$ and coherent $(O)$ sources.

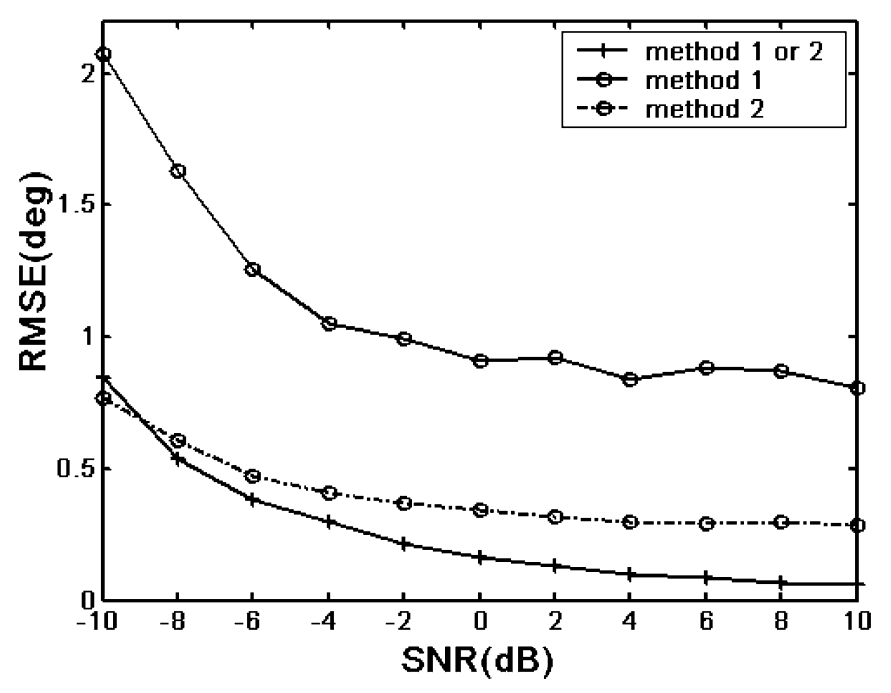

Fig. 2. rmse of the DOA estimates versus input SNR for uncorrelated $(+)$ and coherent $(O)$ sources.

Now we consider the number of sources can be resolved by the three methods for a 9-element uniform linear array. The result is listed in Table I. For simplicity, here we assume that each group contains the same number of coherent sources. It can be seen that the number of sources resolved by our methods can be greater than the number of array elements.

In the first simulation, we consider one uncorrelated source coming from $-25^{\circ}$ and a group of three coherent sources coming from $\left[42^{\circ},-12^{\circ}, 5^{\circ}\right]$. The fading amplitudes and phases of the coherent sources are $[1,0.8,0.7]$ and $\left[92.79^{\circ}, 38.07^{\circ}, 170.28^{\circ}\right]$, respectively. Assume that all source signals are of equal power. The rmse of the DOA estimates versus input SNR is shown in Fig. 1. Note that the same method is used for the uncorrelated sources in Methods 1 and 2. The figure illustrates that the estimates by our new methods are considerably more accurate than that by Method 0, especially when the SNR is relatively low. This is because that our deflation approach uses the whole array to estimate the uncorrelated sources and then uses an equivalently reduced array to estimate 
the coherent sources in a subsequent stage without the interference of the uncorrelated sources, while Method 0 estimates the DOAs of all the uncorrelated and coherent sources simultaneously based on the reduced array. Fig. 1 shows also that Method 2 outperforms Method 1 slightly.

The second simulation considers the scenario that the total number of sources exceeds the number of array elements. There are six uncorrelated sources coming from $\left[-25^{\circ}, 0^{\circ}, 10^{\circ}, 30^{\circ}, 52^{\circ},-45^{\circ}\right]$ and a group of four coherent sources coming from $\left[42^{\circ},-12^{\circ}, 5^{\circ}-40^{\circ}\right]$. The fading amplitudes and phases of the coherent sources are $[1,0.8,0.7,0.6]$ and $\left[92.79^{\circ}, 38.07^{\circ}, 170.28^{\circ}, 248.75^{\circ}\right]$, respectively. Assume that all sources are of equal power. Method 0 will fail in such scenario, while as shown in Fig. 2 our two deflation approaches still have good estimation performance. Similar to the first simulation, Method 2 has a better performance than Method 1.

\section{CONCLUSION}

In this letter, we have proposed a novel deflation approach to DOA estimation for symmetric uniform linear array when both uncorrelated sources and coherent sources are presented. Two methods for deflation are developed. The number of sources resolved by our deflation approach can be greater than the number of array elements, with an upper limit of $M-P-1$ uncorrelated sources plus $\lfloor(M-1) / 2\rfloor$ coherent sources in $P$ groups. Simulation results validate the effectiveness of our deflation approach and illustrate that our approach outperforms the existing method proposed in [5]. Our approach can also be extended to the scenario where the sources can be either uncorrelated, or partially correlated, or coherent.

\section{REFERENCES}

[1] S. U. Pillai and C. S. Burrus, Array Signal Processing. New York: Springer-Verlag, 1989.

[2] L. C. Godara, "Application of antenna arrays to mobile communications, part ii: Beam-forming and direction-of-Arrival considerations," Proc. IEEE, vol. 85, pp. 1195-1245, Aug. 1997.

[3] R. O. Schmidt, "Multiple emitter location and signal parameter estimation," IEEE Trans. Antennas Propag., vol. 34, pp. 276-280, Mar. 1986.

[4] S. U. Pillai and B. H. Kwon, "Forward/backward spatial smoothing techniques for coherent signal identification," IEEE Trans. Acoust., Speech, Signal Process., vol. 37, pp. 8-15, Jan. 1989.

[5] F. M. Han and X. D. Zhang, "An ESPRIT-Like algorithm for coherent doa estimation," IEEE Antennas Wireless Propag. Lett., vol. 4, pp. 443-446, 2005.

[6] R. T. Behrens and L. L. Scharf, "Signal processing applications of oblique projection operators," IEEE Trans. Signal Process., vol. 42, pp. 1413-1424, Jun. 1994.

[7] M. L. McCloud and L. L. Scharf, "A new subspace identification algorithm for high resolution doa estimation," IEEE Trans. Antennas Propag., vol. 50, pp. 1382-1390, Oct. 2002. 